\title{
Introduction to the Co-development with Software and Business Engineering Frameworks and Methods Mini-Track
}

\author{
Tomi Dahlberg \\ Turku School of Economics \\ at University of Turku \\ FIN-20014 University of Turku \\ Turku, Finland \\ tomi.dahlberg@utu.fi
}

\author{
Altti Lagstedt \\ Haaga-Helia University of Applied \\ Sciences, \\ Ratapihantie 13 FIN-00520 Helsinki \\ Helsinki, Finland \\ altti.lagstedt@haaga-helia.fi
}

\author{
Robert Winter \\ Institute of Information Management, \\ University of St. Gallen \\ Mueller-Friedberg Strasse 8 \\ CH-9000 St. Gallen, Switzerland \\ robert.winter@unisg.ch
}

The history of information systems (IS) development frameworks, methods and tools exceeds 60 years. New or improved versions of existing frameworks, methods and tools, plan-driven (e.g. waterfall) and change-driven (e.g. agile), are launched continuously. Despite of this, the success rate of IS development projects has remained at the modest 35-40\% level with only a slight $10-15 \%$ success rate increase compared to the $25-30 \%$ level of the mid 1980s. Moreover, a significant amount of IS and other solution development projects are carried out without any development framework, method and tools. The purpose of IS development is not only to design and implement an IS. Within IS development, software development initiatives are embedded in or related to business development initiatives - sometimes focused on a function or product, but increasingly enterprise-wide like digital transformation or even cross-enterprise like digital platforms. As a consequence, software development needs to be integrated with business process development, re-engineering or digitalization/digitization of existing processes; development of new digital products, services or businesses; digitization of existing products, services and businesses; or the development of other types software-enabled incremental and revolutionary innovations. In the resulting activities and projects, it is often necessary to align different kinds of competences, frameworks, methods, tools and even mindsets in order to control and synchronize work done in various subprojects or activities. The modest success rates of IS and other development projects encourage both the academic community and practitioners to search for better answers to the needs of software and business engineering. As a whole, we need to increase our knowledge base and theoretical understanding about the interrelations between software, IS and business development during the planning, development and run (such as maintenance and operation) phases of their lifecycles.

Software and business development projects vary widely regarding their problem or solution complexity, the number of involved persons and actors, their novelty, their context, and / or their importance, just to mention few of the relevant characteristics. Software development could be conducted inhouse, co-sourced or outsourced. In addition to that, the participation of internal and external business professionals, software users, internal and external IT professionals may change during the lifecycle of software and business development from requirements engineering to IS/software roll-outs and the post-evaluation of an IS/software. As a consequence, an IS/software user organization may not be able or willing to assess all possibilities and limitations of the various software development methods.

To overcome the above described limitations of conceptual and theoretical understanding, the goal of this mini-track, organized for the first time at HICSS 2021 , is to offer research contributions that open up new perspectives and insights for better co-development with software and business engineering frameworks, methods and tools. In this year's mini-track, two papers were accepted. They offer representative overviews to the themes of the mini-track. Both contributions were selected after extensive peer reviews with minor suggestions for revisions.

In their paper Fit to Context Matters - Selecting and Using Information Systems Development Methods to Develop Business in Digitalization Contexts, the minitrack co-chairs Dahlberg and Lagstedt outline a research agenda and propositions for research between the three layers of digitalization: software IS and business. The authors contemplate these issues on the basis of their prior published studies and new empirical data.

The second paper Performance Measurement in Scaled Agile Organizations by Korpivaara et al. investigates with an exploratory case study method, how agile organizations set performance objectives and metrics as well as balance between business and software engineering goals. The paper offers performance measurement dimensions to guide the selection and prioritizing of performance objectives in scaled agile organizations. 